\title{
Expression patterns of the homeo box- containing genes En-1 and En-2 and the proto-oncogene int-1 diverge during mouse development
}

\author{
Claytus A. Davis and Alexandra L. Joyner \\ Division of Molecular and Developmental Biology, Mount Sinai Hospital Research Institute and the Department of Medical
} Genetics, Toronto, Ontario, Canada, M5G-1X5

\begin{abstract}
We have compared the expression of the murine genes En-1,En-2, and int-1 during development by in situ hybridization. Expression of all three genes was first detected at 8.0 days in overlapping bands of the anterior neural folds. By 12.0 days the expression patterns diverged. En-1 and En-2 were expressed in a similar ring of cells in the central nervous system (CNS) at the midbrain/hindbrain junction. En-1 was also expressed de novo in two lateral stripes extending the length of the hindbrain and spinal cord, in the developing vertebral column, in two lateral stripes of dermatome-derived cells, and in the tail and limb buds. By 12.0 days int-1 expression showed little overlap with the En genes and could not be detected at later stages. At 15.5 days En gene expression was primarily limited to the midbrain/hindbrain in overlapping but nonidentical sets of differentiated cells. In the adult, En-1 and En-2 marked the same sets of cells in the pons, but En-2 alone was detected in the granular layer of the cerebellum. The results are consistent with int-1 and the En genes playing a role early in development in defining spatial domains in the CNS. Later in development the En genes may have an additional function in neurogenesis. En-1 expression in the developing pericordal tube suggests that it may also be involved in vertebral assembly.
\end{abstract}

[Key Words: Homeo box; engrailed; int-1; RNA in situ; mouse development; brain; vertebrae]

Received August 31, 1988; revised version accepted November 10, 1988.

The ease with which mutants may be isolated and characterized in Drosophila melanogaster has led to the isolation and study of a large number of genes controlling pattern formation in development. One conserved sequence motif that is both unique to these genes and prevalent among them is the homeobox. Compared with Drosophila, knowledge of the genetic control of development in vertebrates has been slow to accumulate due to the difficulty of directly identifying and mutating the genes involved. However, many genes potentially important in vertebrate development, for instance, the homeo box-containing genes (e.g., the Hox genes; for review, see Fienberg et al. 1987; Holland and Hogan 1988a), have been isolated by using Drosophila developmental genes as probes.

We have been studying the two murine genes, En-1 and En-2, which were isolated on the basis of their extensive sequence homology to the Drosophila segmentation gene engrailed (en) (Joyner et al. 1985; Joyner and Martin 1987). Our in situ hybridization analysis of the expression of En-2 during development has shown that it is expressed early in embryogenesis in a band of cells across the anterior neural folds and continues to be ex- pressed in the same region of the developing and adult brain (Davis et al. 1988). Northern blot analysis of expression of En-1 in embryo tissues showed that its expression pattern is not restricted to the brain but can also be detected in the spinal cord, limbs, and carcass (Joyner and Martin 1987).

In Drosophila, mutational analysis has shown that en is crucial for generation of the posterior compartments of each segment (Garcia-Bellido and Santamaria 1972; Lawrence and Morata 1976; Kornberg 1981). en and a related gene, invected (inv), are co-expressed early in embryogenesis in the cells of the posterior compartments of the segments (Dinardo et al. 1985; Fjose et al. 1985; Kormberg et al. 1985; Coleman et al. 1987) and later in development in the developing central nervous system (CNS) (DiNardo et al. 1985; Coleman et al. 1987). The regulation of expression of en in Drosophila is complex. One of the genes involved in maintenance of expression of $e n$ and which also may be capable of initiating its de novo expression is the segment polarity gene wingless $(w g)$ (DiNardo et al. 1988). $w g$ is expressed in a stripe of cells anterior to the en/inv-expressing posterior compartment cells in the cellular blastoderm (Baker 1987; 
Rijsewijk et al. 1987). Recently it has been shown that wg is the Drosophila homolog of the murine oncogene int-1 (Rijsewijk et al. 1987). In the mouse, the expression of int-1 has been shown by in situ analysis to be limited to the CNS during embryogenesis (Wilkinson et al. 1987) and the testes in the adult (Jackobovits et al. 1986; Shackleford and Varmus 1987).

Considering that the two related genes en and inv are co-expressed in the same cells during embryogenesis, it was of interest to determine the pattern of expression of mouse En-1 during development and to compare it to that of the related gene En-2. Furthermore, because in Drosophila wg plays a role in the regulation of expression of at least $e n$ and is expressed at the cellular blastoderm stage adjacent to the en/inv-expressing cells, it was important to determine whether the expression patterns of En-1, En-2, and int-1 suggest a similar interaction between these genes. In this paper, we have used in situ hybridization to examine the pattern of expression of En-1 at stages throughout development and to compare the expression patterns of En-1,En-2, and int-1. These studies demonstrate that expression of the three genes begins within the same 6-hr period of early somite formation and is restricted to similar overlapping domains within the neural folds. By 12 days of embryonic development, however, the patterns of expression of these three genes have greatly diverged and any overlap in expression is restricted to a small region of the metencephalon. Also, although both en genes are expressed in similar regions of the developing brain, En-1 is also expressed transiently at midgestation in the spinal cord, in the limb and tail buds, and in two somite-derived tissues.

\section{Results}

\section{Experimental strategy}

To analyze the expression of En-1,En-2, and int-1 in situ during mouse embryogenesis, we used SP6 transcripts of small regions of the three genes. The En-2 probe was the same 250-bp fragment of $3^{\prime}$ untranslated cDNA that was used previously (Davis et al. 1988). For En-1, a 180-bp 3' untranslated cDNA fragment was used. This probe hybridized to a single copy sequence on Southern blots of mouse genomic DNA. In a Northern blot analysis of mouse embryo total RNA, it hybridized to only the major 2.8 -kb En-1 transcript in all of the tissues examined including RNA from 9.5- and 12.5-day embryos and 16.5-day brain or body without the head (C. Logan and A.L. Joyner, data not shown). The signal was reduced by at least 10-fold in the 16.5-day body RNA compared to the brain. Thus, this probe appears to detect a single En-1 transcript. For int-1 a 230-bp EcoRI-ApaI fragment derived from the int-1 probe, used previously by Wilkinson et al. (1987), was used. The negative sense strand probes were made from the same fragments cloned in the complementary vector so that in all cases the probes were generated by transcription from the SP6 promoter. Wherever possible, adjacent sections were probed with each of the three antisense probes. Sense-strand negative control probes were included in each series.

Whole embryos for four different stages of gestation, from 7.7 to 15.5 days, as well as 17.5-day and adult brain were examined. When the sense strand-negative control probes were used, occasional artifactual binding occurred near the borders of the sections or lumens within the embryos. Nonspecific binding was also consistently observed around the edges of the cerebellum as well as to the dentate gyrus and pyramidal cell layer of the adult hippocampus.

Expression of En-1, En-2, and int-1 was first detected in overlapping domains during formation of the early somites

In our previous in situ study we were first able to detect En-2 transcripts in outbred CD-1 mice during a 6-hr period covering the formation of the first six somites around day 8.0 (Davis et al. 1988). Similarly, the earliest detectable expression of int-1 by in situ analysis was between days 8 and 9 in CBA mice (Wilkinson et al. 1987). This time corresponds to 7.5-8.5 days in other mouse strains, including CD-1.

To determine the degree of coincidence of expression of En-1, En-2, and int-1, 7.7-day (CD-1 outbred mice) embryos which possessed a neural plate and foregut pocket, but lacked any somites, and 8.0-day embryos which had between 4 and 6 somites were hybridized in situ to the probes specific for each of the genes. The 7.7day embryos showed no hybridization specific to any of the probes (C.A. Davis, data not shown). However, by 8.0 days all three probes hybridized to the embryos (Fig. 1). En-1 and En-2 transcripts were only detected in a band of the neural folds at the level of the foregut pocket. Although it is not possible from this analysis to be certain that the two expression patterns are identical, they are very similar and the slight variations we observed could be due to slight differences between neighboring sections. The int-1 probe gave a higher background than the En probes in all tissues from all stages examined. Nevertheless, from examining six embryos in sagittal or cross sections, it was clear that the same region of the neural folds that hybridized to the En probes was also the major site of int-1 expression in the 8.0-day embryos (Fig. 1b). However, the band of int-1 expression was not as broad as that of the en genes and appeared to be contained within the band of $e n$ expression at the rostral side. In addition, the lateral edges of the neural plate caudal to the int 1 band of expression in the neural folds also hybridized (data not shown). The int-1 expression was the same as that observed by Wilkinson et al. (1987).

The expression patterns of En-1, En-2 and int-1 have diverged by 12.0 days

Expression within the CNS By 12.0 days the neural folds have joined to form the neural tube, and large morphological changes in the brain make it possible to identify the regions from which adult brain structures will 

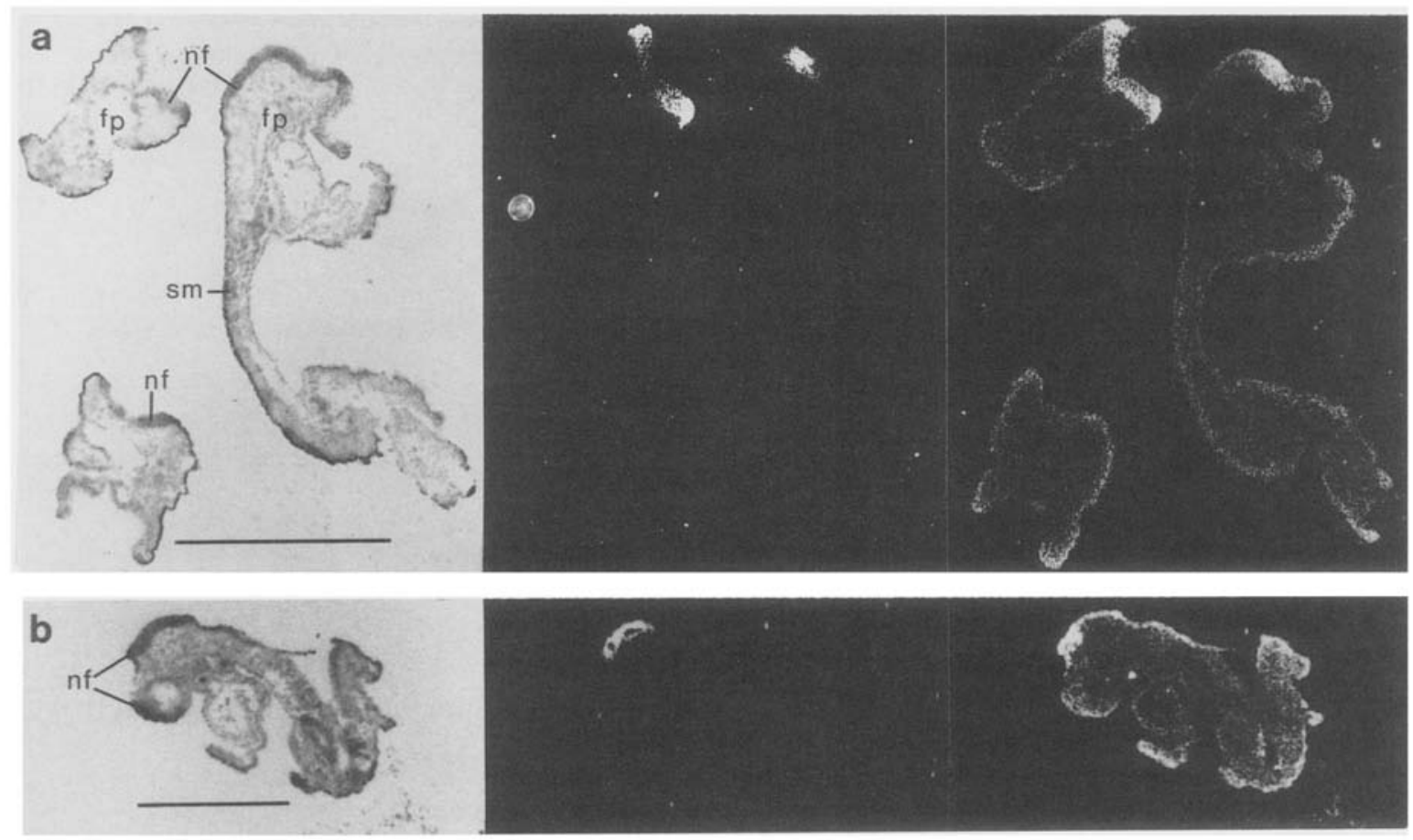

Figure 1. Localization of En-1, En-2, and int-1 transcripts in sagittal and transverse sections of 8.0-day embryos. (Left) Bright-field illumination to show morphology; (right) dark-field illumination to show the hybridization to the different probes. $(a)$ Sagittal $(r i g h t)$ and two transverse sections: one at the level of the foregut pocket (top) and the other just caudal to the pocket (bottom). Dark-field pictures show En-2 (left) and En-1 (right) hybridization. (b) Parasagittal section. Dark-field pictures show En-2 (left) and int-1 (right) hybridization. (fp) Foregut pocket; (nf) neural fold; $(\mathrm{sm})$ somite. Scale bars represent $1 \mathrm{~mm}$.

develop. Neurogenesis has also begun and differentiated neurons and glioblasts have started to migrate out of the germinal zone. By day 12.0 , this process is well under way in the spinal cord and hindbrain and, to a lesser extent, in the midbrain.

As was shown previously (Davis et al. 1988), En-2 was expressed at high levels throughout a ring of the neural tube at the midbrain/hindbrain junction (Figs. 2a,b and $3 \mathrm{c}$, d), a region that will later form the pons, cerebellum, and part of the colliculi and periaqueductal gray. This region is at the same level of the CNS as the band of expressing cells observed at 8.0 days and probably consists of their descendants. En-1 was expressed in the same region of the developing brain (Figs. 2a,b and $3 \mathrm{~b}-\mathrm{d})$, although there were some differences. Within the germinal zone En-1 expression was diminished compared to En-2 in the caudal and dorsal regions where the cerebellum, pons, and colliculi later develop (Figs. 2a,b and $3 \mathrm{c})$. However, in the overlying regions of differentiated cells, expression of $E n-1$ was more similar to that of En-2, both in its pattern and intensity (Figs. 2a and $3 \mathrm{c})$.

In addition to the domain of En-1 expression in the developing midbrain/hindbrain, it was also expressed in two ventrolateral stripes extending from the rostral hindbrain down the length of the spinal cord (Figs. 2b,c and $3 b-d)$. Here, both germinal zone and differentiated cells expressed the gene. However, in contrast with the germinal zone expression of $E n-1$ in the midbrain, where the entire thickness of the layer predominantly hybridized, the cells nearest the lumen in the stripe did not express En-1 (Fig. 3d). Since we did not detect hybridization of the En-1 probe to the 8.0-day neural plate anywhere other than the headfold band, the expression in the 12-day spinal cord almost certainly represents a second initiation of En-1 expression in the CNS.

Although int-1 was expressed in the same domain of the CNS as En-1 and En-2 at 8.0 days, the hybridization pattern we observed at 12.0 days was very different. Hybridization occurred primarily in a thin stripe of cells along the dorsal midline of the spinal cord and brain (Figs. 2c and 3c) and outlined the edge of the membranous roof of the hindbrain (C.A. Davis, data not shown). In the study by Wilkinson et al. (1987), int-1 expression at 12.5 days was also observed in several small regions of the midbrain, which we did not see. Perhaps int-1 expression in the midbrain is punctate and the slides we examined did not contain the expressing cells. Therefore, the only overlap in expression of the en and int-1 genes that we observed at 12.0 days was where the expression of int-1 traversed the dorsal region of en expression in the brain (Fig. 3c).

Expression outside the CNS En-2 showed very limited expression outside the CNS. Previously, we described faint hybridization in the region of Rathke's pouch (Davis et al. 1988). This second analysis confirmed that cells around Rathke's pouch expressed En-2 (Fig. 3d). We 

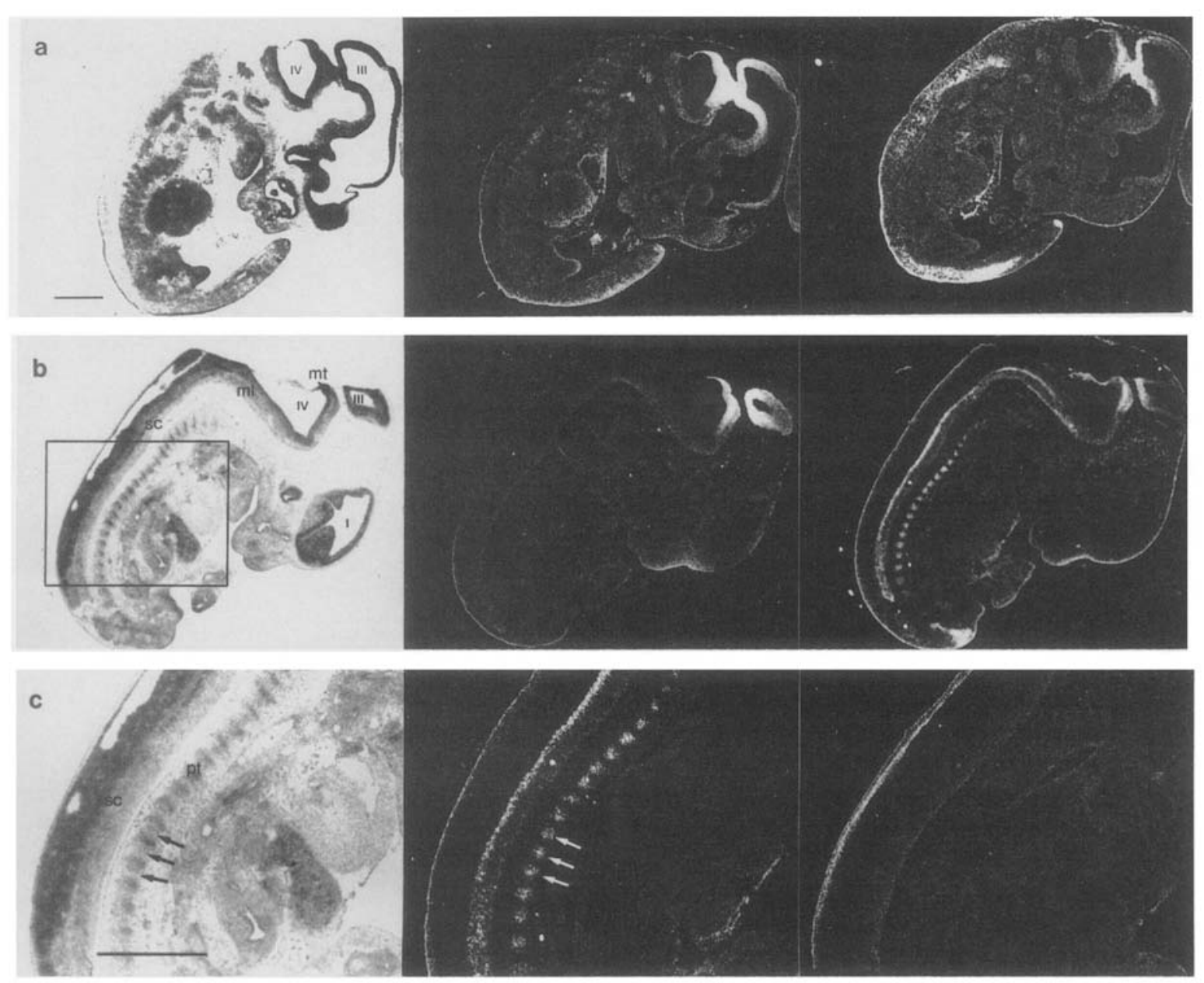

Figure 2. Localization of En-1,En-2, and int-1 transcripts in sagittal sections of a 12.0-day embryo. $(a, b)$ Parasagittal sections. Darkfield pictures show En-2 (left) and En-1 (right) hybridization. (c) Higher magnification of the region in the box in b. Dark-field pictures show En-1 (left) and int-1 (right) hybridization. Arrows point to the loose-packed sclerotome-derived cells of the pericordal tube. (ml) Myelencephalon (hindbrain); (mt) metencephalon; (pt) pericordal tube; (sc) spinal cord; (I) first ventricle; (III) third ventricle; (IV) fourth ventricle. Scale bars represent $1 \mathrm{~mm}$.

do not know the origin or fate of this tissue. In contrast, En-1 was expressed in a number of other tissues, two of which were of somite origin. En-1 was expressed in a swath of dermatome-derived cells extending from the top of the head to the tip of the tail on both sides of the embryo (Figs. $2 \mathrm{a}$ and $3 \mathrm{~b}-\mathrm{d}$ ). The second tissue of somite origin that expressed En-1 was the sclerotome-derived loosely packed cells of the pericordal tube (Fig. 2b,c), which later gives rise to the vertebrae. As these cells alternate with the densely packed cells from which the intervertebral disks form (Fig 2c), the developing backbone showed a periodic or striped pattern of expression of En-1 throughout the length present on the sections we examined, extending from the thoracic to tail prevertebrae. Additional En-1 expression was detected in the tail bud (Fig. 2a,b) and regions of the limb buds (Fig. 3c,d).

\section{Following neurogenesis En-1 and En-2 mark} overlapping but nonidentical groups of cells in the brain

By 15.5 days it was apparent that En-1 and En-2 expression was mainly limited to the brain. We could not de- tect any expression of int-1 at 15.5 days or later, although lower levels could have been masked by background. Outside of the brain no hybridization of the En-2 probe could be seen to the region around Rathke's pouch. Some expression of En-1 could be detected in the 15.5-day spinal cord (Fig. 4b) although the hybridization was faint and confined to two narrow stripes. Similarly, faint hybridization could be seen in the vertebrae (Fig. 4b) and under the skin (Fig. 4c,d).

In the 15.5-day brain, the En genes were still strongly expressed in the midbrain/hindbrain region (Fig. 4c,d). By this time, neurogenesis is well underway and by 17.5 days will be almost completely finished in this region. An exception to this is the external granular layer of the cerebellum that forms from migrating germinal zone cells from the rhombic lip at the junction of the hindbrain and midbrain. These cells form a layer coating the developing cerebellum and continue to divide until approximately postnatal day 10 , when they migrate into the interior of the cerebellum to form the granular layer of neurons.

By 15.5 days, it was clear that En-1 and En-2 marked 
Figure 3. Localization of En-1, En-2, and int-1 transcripts in frontal sections of a 12.0day embryo. (a) Bright-field photograph showing structures of the brain. Lines b,c,d indicate the levels of the frontal sections shown in b,c,d, respectively.(b) Dark-field photograph of a frontal section at the level $b$, showing En-1 hybridization. (c) Frontal sections at the level c. Dark-field pictures show En-2 (left), En-1 (middle), and int-1 (right) hybridization. (d) Frontal sections at the level d. Dark-field pictures show En-2 (left) and En-1 (right) hybridization. (gz) Germinal zone-dividing undifferentiated neuroepithelium; (iz) intermediate zone-postmitotic neurons and glioblasts; (ml) myelencephalon; (mt) metencephalon; (pn) pons; (rp) Rathke's pouch; (sc) spinal cord; (III) third ventricle; (IV) fourth ventricle. Scale bars represent $1 \mathrm{~mm}$.
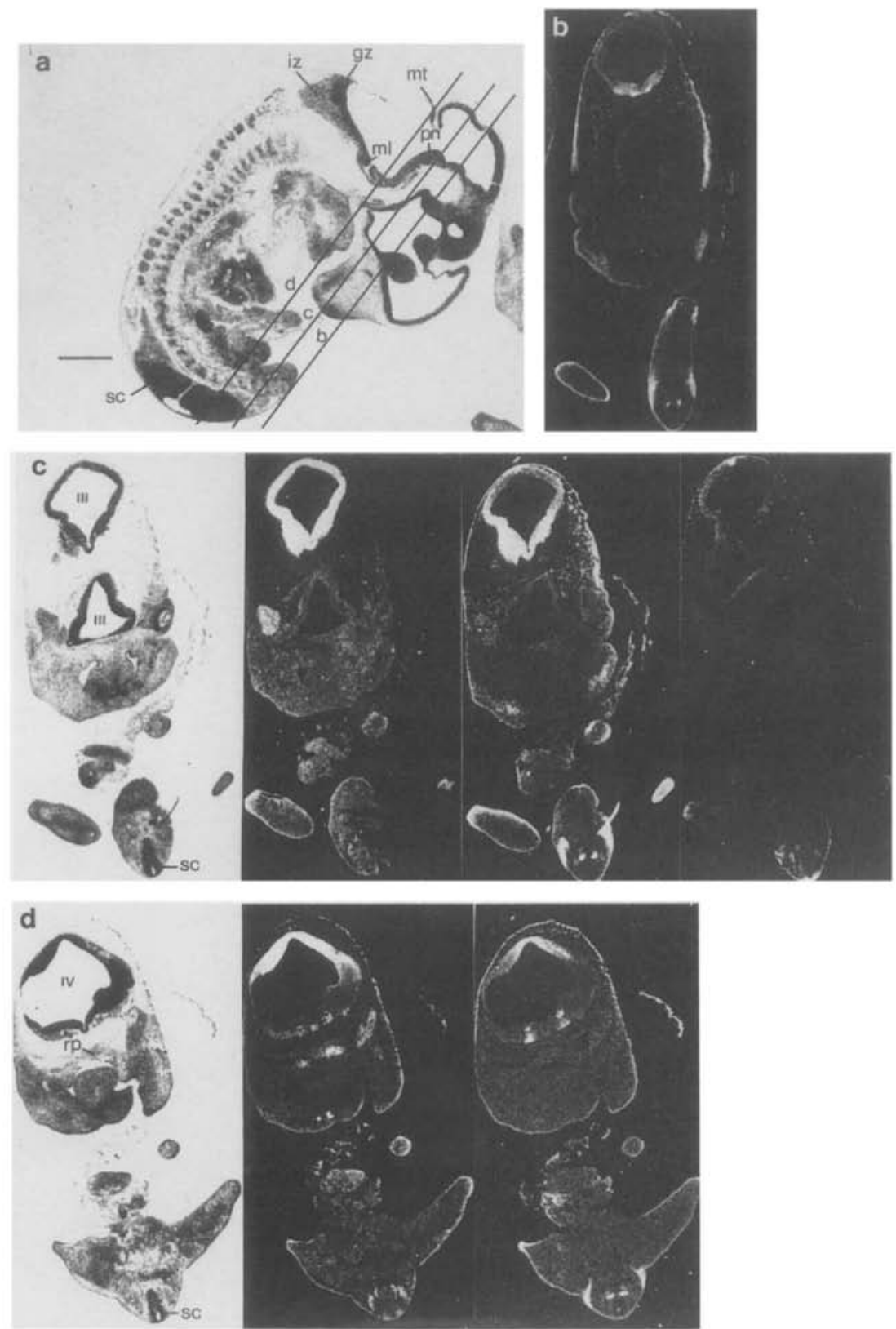

different populations of differentiated cells in the brain outside the germinal zone (Fig. 4c,d). En-1 was expressed in only a few patches of cells in the cerebellum and was weakly expressed in the lateral colliculi, whereas En-2 was expressed extensively in both. In the pons and periaqueductal gray, En-1 and En-2 showed the same hybridization pattern, marking cells extending forward from just under the cerebellum to the midbrain (Fig. 4c). In the hindbrain, the expression of En-1 which appeared at 12.0 days to be contiguous with the spinal cord expression continued in the 15.5-day samples, although it was limited to a ventral patch of differentiated cells (Fig. $4 \mathrm{~d})$.
In the 17.5-day brain sections, En-2 was expressed extensively in the cerebellum, including the external granular layer, whereas $E n-1$ was expressed to a lesser degree (Fig 5). In the pons, En-2, but not En-1, expression was detected in a group of cells just under the caudal edge of the cerebellum (Fig. 5b). This was tentatively identified as the trigeminal motor nucleus. The rest of the pontine hybridization was similar for the two genes and extended from the junction of the cerebellum and pons rostrally into the midbrain. In the hindbrain En-1 was expressed in the same cells described in the 12.0-day and 15.5-day embryos (Fig. 5b).

In the adult brain (Fig. 6), the extent of expression of 

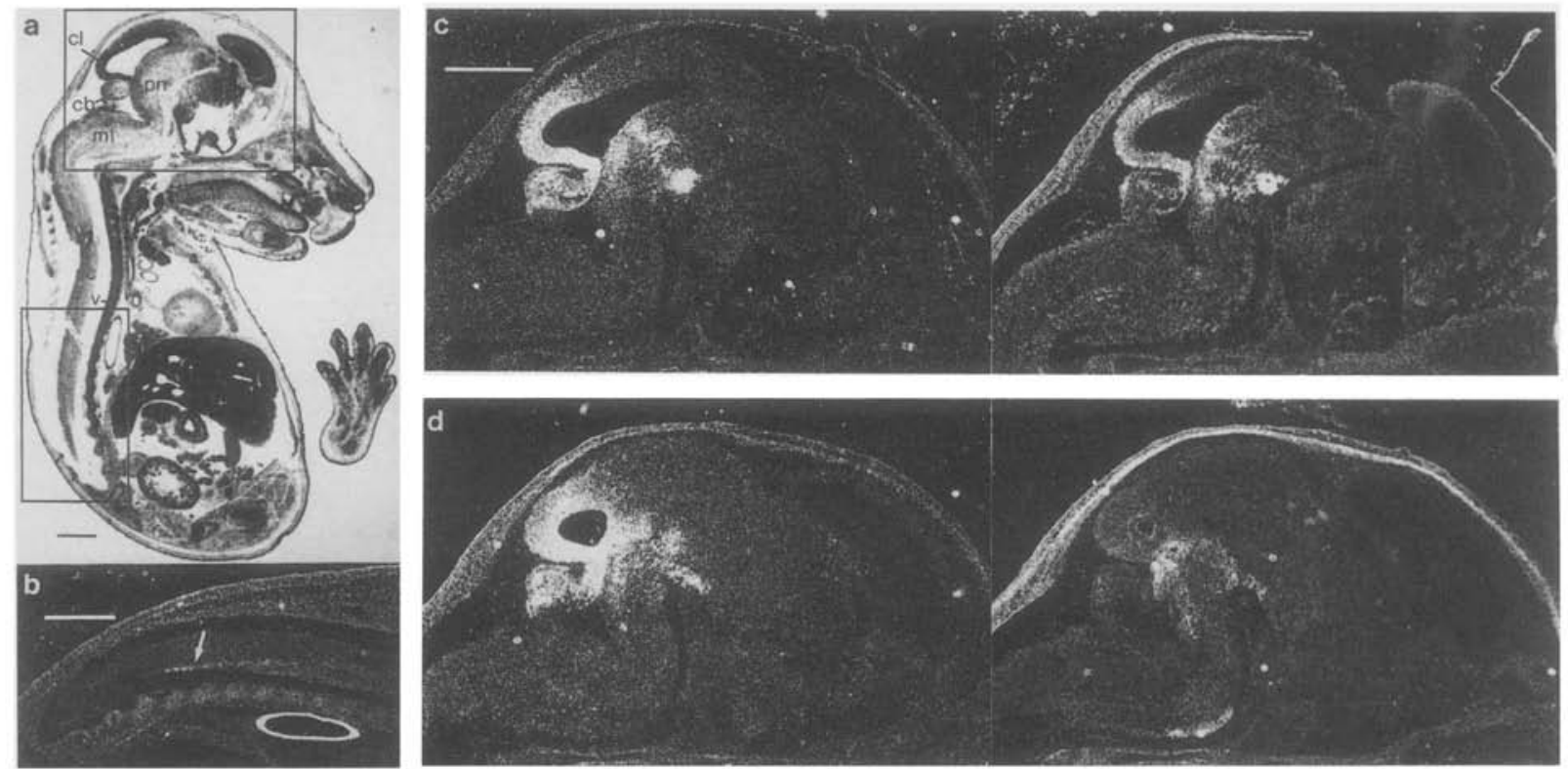

Figure 4. Localization of En-1 and En-2 transcripts in sagittal sections of a 15.5-day embryo. (a) Bright-field photograph showing a midsagittal embryo section. Boxes show the regions magnified in $\mathrm{b}, \mathrm{c}, \mathrm{d}$. (b) Dark-field of the region in the lower box in $a$ showing En-1 hybridization (photograph rotated $90^{\circ}$ clockwise). $(c, d)$ Detail of regions corresponding to the upper box in $a$. Dark-field pictures show En-2 (left) and En-1 (right) hybridization in midsagittal (c) and parasagittal (d) sections of the brain. (cb) Cerebellum; (cl) colliculi and periaqueductal gray; (ml) myelencephalon; $(\mathrm{pn})$ pons; (sc) spinal cord; $(\mathrm{v})$ vertebrae. Scale bars represent $1 \mathrm{~mm}$.

the En genes had decreased further. In the cerebellum, strong En-2 expression was seen in the granular layer, whereas no En-1 expression was detected (Fig. 6b). The binding of the $E n-1$ probe to the Purkinje layer and the exterior surface of the cerebellum (Fig. $6 \mathrm{~b}$ ) is probably artifactual, because it was seen with the negative control probes as well. There was no hybridization of either gene to the dorsal periaqueductal gray or the colliculi. In the pons and ventral periaqueductal gray, the pattern of expression of the two genes appeared to be the same. They were both expressed in groups of cells extending forward from under the rostral portion of the cerebellum to the midbrain. The extent of hybridization diminished rostrally in the same fashion as was seen in the 17.5-day sagittal brain sections. Near the junction of the pons and midbrain, hybridization had diminished to two lateral patches of cells (Fig. 6c). From the caudal to rostral pons we have tentatively identified the hybridizing cells as
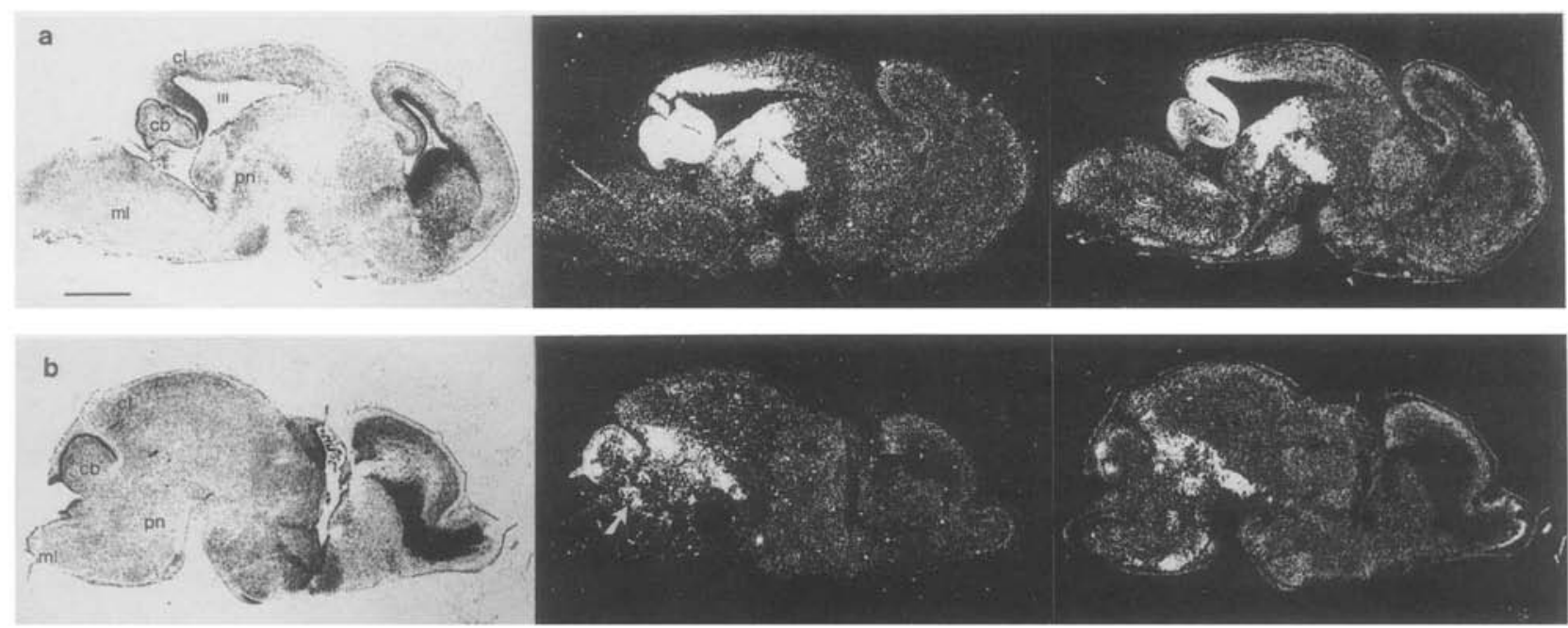

Figure 5. Localization of En-1 and En-2 transcripts in sagittal sections of a 17.5-day brain. Dark-field pictures show En-2 (left) and En-1 (right) hybridization. (a) Midsagittal sections; $(b)$ parasagittal sections. (cb) Cerebellum; (cl) colliculi and periaqueductal gray; (ml) myelencephalon; (pn) pons; (III) third ventricle. Scale bars represent $1 \mathrm{~mm}$. 


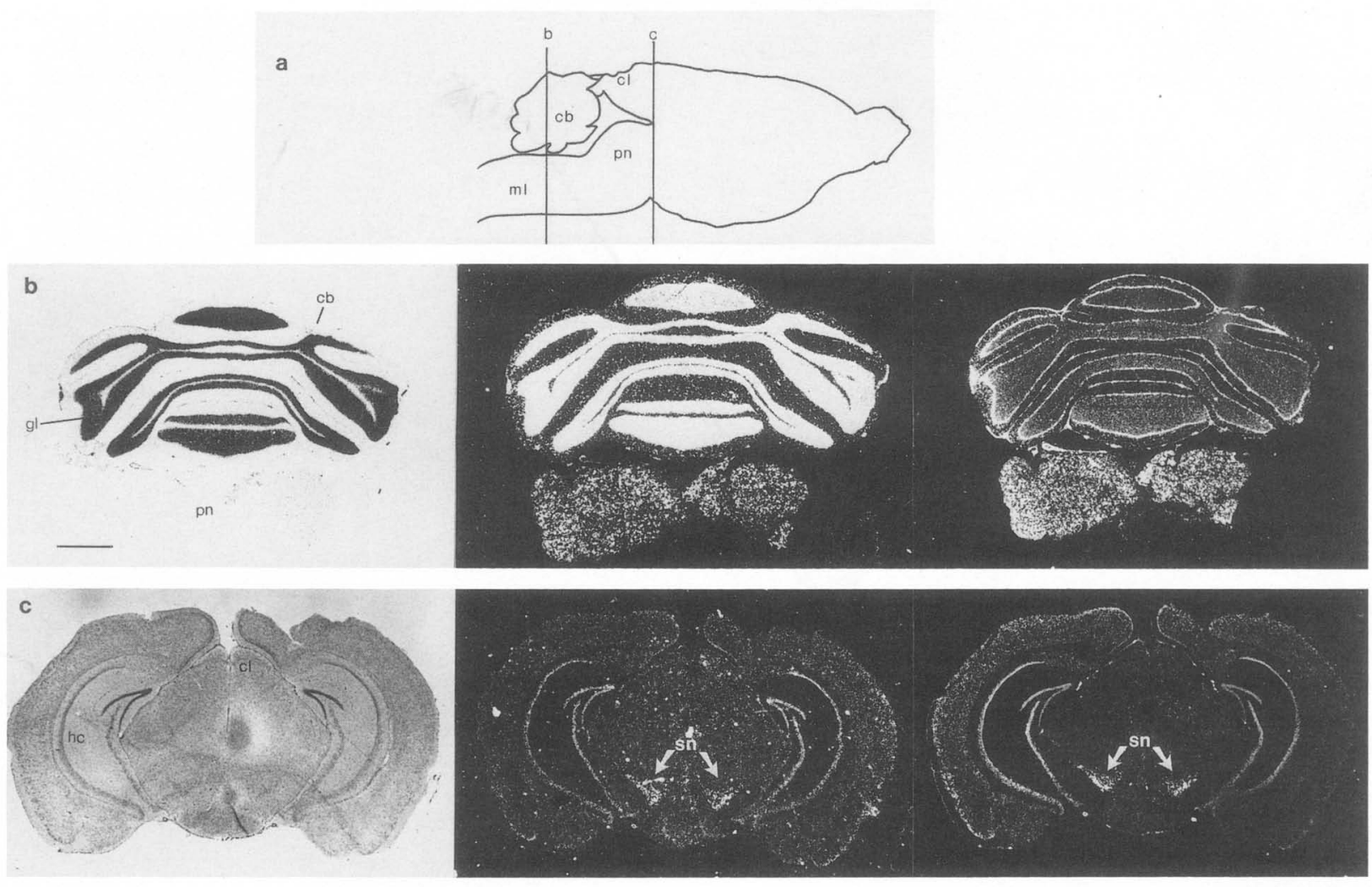

Figure 6. Localization of En-1 and En-2 transcripts in coronal sections of an adult brain. (a) Diagram of a midsagittal adult brain. Lines $\mathrm{b}$ and $\mathrm{c}$ indicate the levels of the coronal sections in $b$ and $c .(b, c)$ Coronal sections at the levels $\mathrm{b}$ and $\mathrm{c}$. Dark-field pictures show En-2 (left) and En-1 (right) hybridization. (cb) Cerebellum; (cl) colliculi; (gl) granular layer; (hc) hippocampus; (ml) myelencephalon; (pn) pons, (sn) substantia nigra. Scale bars represent $1 \mathrm{~mm}$.

belonging to the ventral periaqueductal gray, raphe linearis, interpeduncular nuclei, and substantia nigra. In the sections examined, we did not locate either the trigeminal motor nucleus, which had expressed En-2 in the 17.5-day embryonic pons, or the hindbrain cells, which expressed En-1 earlier.

\section{Discussion}

A comparison of the patterns of expression of En-1,En-2, and int-1 has shown that all three of the genes initiate expression within the same band of the anterior neural folds during formation of the early somites, with additional int-1 expression down the lateral edges of the neural folds. However, by 12.0 days, the overlap between int-1 and the two En genes seemed coincidental. Both En genes continued to express in a band or ring at the midbrain/hindbrain junction, but in our analysis int -1 expression was only observed along the dorsal midline of the spinal cord and brain and was co-expressed with the En genes only where this path crossed the ring of expression of the En genes in the brain. Wilkinson et al. (1987) observed additional hybridization of int-1 to several groups of cells in the midbrain, only some of which would likely be within the region of En expression. Overall, it therefore seems that unlike the situation with $e n$ and wg in Drosophila, the expression data provide little evidence for the regulation of the murine En genes by int-1, although the initial extensive overlap in the expression of the genes is consistent with a regulatory interaction.

By 12.0 days, the expression patterns of $E n-1$ and $E n-2$ have also partially diverged in the midbrain/hindbrain region. The expression of En-1 was weaker than En-2, especially in the germinal zone, although the two genes marked similar sets of differentiated cells. Later in development, the expression of the two genes occurred in overlapping but nonidentical sets of differentiated cells in the brain. Both genes were expressed in the same sets of cells in the periaqueductal gray and pons into adulthood. However, En-2 was expressed in the cerebellum throughout its development and in the cells of the granular layer in the adult, whereas En-1 expression was detected in only a few patches of cells in the cerebellum during development and not at all in the adult.

The most striking difference between $E n-1$ and $E n-2$ was the extensive expression of En-1 outside the midbrain/hindbrain junction at mid-gestation. At 12.0 days En-2 expression was observed only in cells surrounding Rathke's pouch, whereas En-1 was expressed in several other tissues. Within the CNS, En-1 was expressed in two lateral stripes of cells down the length of the hind- 
brain and spinal cord. Outside the CNS a swath of cells running the length of the embryo under the skin and the loosely packed cells of the prevertebral column both expressed En-1. The former is derived from the dermatome and the latter from the sclerotome portion of the somites. In addition to these, the limb and tail buds also expressed En-1. By 15.5 days expression of En-1 was barely detectable outside the CNS. The divergent patterns of expression of $E n-1$ and $E n-2$ differ from their Drosophila counterparts en and inv, which share the same expression pattern throughout development (Coleman et al. 1987).

The initial expression of all three genes may be relevant to the question of compartmentalization of the early CNS. The near simultaneous initiation of expression of the genes at 8.0 days in specific regions of the CNS suggests that, despite their uniform appearance, the neural folds are being subdivided into different regions, at least on the basis of gene expression. It is also during this time that Hox-1.5 has been shown to mark a specific region of the CNS (Gaunt, 1987), although a more widespread hybridization was seen earlier in the entire posterior region of the embryo. As discussed by Nieuwkoop (1985), studies in several amphibian species suggest that there is a subsequent regional commitment in the CNS following the formation of the neural plate. It is conceivable that the En and int-1 genes, as well as other homeo box-containing genes, are involved in this process.

The differences in the later expression of $E n-1$ and En-2 in the CNS support the hypothesis that the genes may also be involved in neural differentiation. Although the genes initially appear to delineate similar or identical regions of the CNS, they later mark overlapping but different groups of differentiated cells. En-2 alone marks what may be the trigeminal motor nucleus in the developing brain, as well as the granular layer of the adult cerebellum. The expression of En-1 in two ventrolateral stripes running the length of the spinal cord and hindbrain at 12.0 days suggests a second independent induction of expression sometime between 8.0 and 12.0 days. Within these stripes, En-1 expression was found in only the outer germinal zone and differentiated cells, consistent with postmitotic cells initiating expression of the gene. Mitosis occurs at the luminal surface of the germinal zone, and cell bodies migrate away from the lumen after mitosis. Thus, postmitotic neurons and/or glioblasts may be initiating expression of $E n-1$ after their last mitosis at the lumen surface.

A dorsal-to-ventral variation in expression within the spinal cord, as is seen with $E n-1$, has also been observed for several Hox genes. Hox-1.4 and Hox-2.5 are expressed primarily in the dorsal spinal cord (Fienberg et al. 1987; Toth et al. 1987), whereas Hox-3.1 is expressed in the ventral spinal cord (LeMouellic et al. 1988). En-1 would define an intermediate zone between these two regions. This division into three different dorsal/ventral regions is suggestive because the spinal cord is classically divided into three portions - the dorsal horn, the intermediate gray, and the ventral horn, which primarily consist of sensory neurons, interneurons, and motor neurons respectively. However, as of yet there is no evidence that the boundaries of gene expression correspond to these functional boundaries, and in the case of Hox-2.1, which also shows a lesser degree of dorsal/ventral restriction in expression, the investigators state that the boundary of expression does not correspond to any functional boundary (Holland and Hogan, 1988b).

An interesting feature of the expression of En-1 is that it is expressed extensively, as are the Hox genes, in mesodermal tissue. However, the expression of $E n-1$ in the somite-derived tissue was quite different than that observed for the Hox genes. The Hox genes are often expressed in a continuous subset of somites and somite derived tissues, whereas $E n-1$ was expressed in a periodic fashion throughout the length of the pericordal tube and in two stripes of dermatome-derived cells running the entire length of the embryo. Its expression within the pericordal tube appears to be the exact complement of the paired box-containing gene Pax1, which is expressed only in the tightly packed cells of the pericordal tube; cells that will later form the invertebral disks (Deutsch et al. 1988). Both genes may be expressed during the same period of development, with En-1 beginning between 8 days and 12 days and decreasing by 15 days and Pax 1 beginning around 9 days and dropping sharply between 15 and 16 days. Thus, it is tempting to speculate that in addition to its role in the CNS, En-1 may be involved in coordinating vertebrae assembly.

\section{Methods}

\section{Sample preparation}

Embryos from outbred CD-1 mice (Charles River) were collected between 7.5 and 17.5 days of gestation and removed from the uterus /the midpoint of the dark interval during which plugging occurred was considered day 0). The samples were then frozen, sectioned, and stored, as described previously ( $\mathrm{Da}$ vis et al. 1988).

\section{Preparation of probes}

To generate suitable probes for the in situ analysis restriction fragments from cDNA clones of the genes $E n-1, E n-2$, and int-1 were subcloned into the SP6 transcription vectors pGEM-1 and pGEM-2. For En-2 the $3^{\prime}$ noncoding BgII-SstI fragment described previously was used (Davis et al. 1988). For En-1, a 180bp Sau3A-EcoRI fragment of 3' untranslated DNA was used. The 5' $\sim 900$-bp EcoRI fragment from the cDNA clone $\lambda$ En-1c (Joyner and Martin 1987) was gel-purified and digested with Sau3A, and the 180-bp 3' Sau3A-EcoRI fragment subcloned into BamH-EcoRI-cut pGEM1 and pGEM2 plasmids. For int-1 a 230-bp EcoRI-ApaI fragment from the cDNA fragment used in a previous in situ study was used (Wilkinson et al. 1987). Sense and antisense transcripts in corporating ${ }^{35} \mathrm{~S}$ were made from the SP6 promoter, as described previously (Davis et al. 1988). The quality of the probes was checked by gel electrophoresis before use.

\section{In situ hybridization to tissue sections}

Hybridizations were done as described previously using a final probe concentration of $0.2 \mu \mathrm{g} / \mathrm{ml} \cdot(\mathrm{kb}$ of complexity) for $15-24$ 
$\mathrm{hr}$ at $39^{\circ} \mathrm{C}$. The washed and dipped slides were exposed for $\sim 30$ days. The sections were stained with toluidine blue and photographed using a Leitz macroscope.

\section{Acknowledgments}

First and foremost, we would like to thank Dr. Janet Rossant for her assistance throughout the experimental work and her critical review of the manuscript. We are grateful to Drs. David Wilkinson and Andrew McMahon for the gift of the int-1 cDNA clone and to Cairine Logan for the Northern blot containing mouse embryo RNA. We would also like to thank Dr. Derek van der Kooy and Leslie Krushel for their assistance in analyzing the expression patterns and Dr. Inna Gitelman for her assistance with the manuscript. This work was supported by a grant from the Medical Research Council (MRC) of Canada (A.J.) and the Natural Sciences and Engineering Research Council of Canada (A.J.). A.J.is an MRC scholar, and C.A.D. was supported by an MRC studentship.

\section{Notes}

Since submitting this manuscript, a paper has appeared describing the early expression of $E n-1$ in mouse embryos which is in agreement with the results presented here (D. Davidson et al., Development 104: 305-316).

\section{References}

Baker, N.E. 1987. Molecular cloning of sequences from wingless, a segment polarity gene in Drosophila: The spatial distribution of a transcript in embryos. EMBO /. 6: 1765-1773.

Coleman, K.G., S.J. Poole, M.P. Weir, W.C. Soeller, and T. Kornberg. 1987. The invected gene of Drosophila: Sequence analysis and expression studies reveal a close kinship to the engrailed gene. Genes Dev. 1: 19-28.

Davidson, D., E. Graham, C. Sime, R. Hill. 1988. A gene with sequence similarity to Drosophila engrailed is expressed during the development of the neural tube and vertebrate in the mouse. Development 104: 315-316.

Davis, C.A., S.E. Noble-Topham, J. Rossant, and A.L. Joyner. 1988. Expression of the homeo box-containing gene En-2 delineates a specific region of the developing mouse brain. Genes Dev. 2: 361-371.

Deutsch, U., G.R. Dressler, and P. Gruss. 1988. Pax-1, a member of a paired box homologous murine gene family, is expressed in segmented structures during development. Cell 53: 617-625.

DiNardo, S. J.M. Kuner, J. Theis, and P. O'Farrell. 1985. Development of embryonic pattern in $D$. melanogaster as revealed by accumulation of the nuclear engrailed protein. Cell 43: 59-69.

DiNardo, S., E. Sher, J. Heemskerk-Jongens, J.A. Kassis, and P.H. O'Farrell. 1988. Two-tiered regulation of spatially patterned engrailed gene expression during Drosophila embryogenesis. Nature 332: 604-609.

Fienberg, A.A., M.F. Utset, L.D. Bogarad, C.P. Hart, A. Awgulewitsch, A. Ferguson-Smith, A. Fainsod, M. Rabin, and F.H. Ruddle. 1987. Homeo box genes in murine development. Curr. Topics Dev. Biol. 23: 233-256.

Fjose, A., W.J. McGinnis, and W.J. Gehring. 1985. Isolation of a homoeo box-containing gene from the engrailed region of Drosophila and the spatial distribution of its transcripts. Nature. 313: 284-289.

Garcia-Bellido, A. and P. Santamaria. 1972. Developmental analysis of the wing disc in the mutant engrailed of Drosophila melanogaster. Genetics 72: 87-104.
Gaunt, S.J. 1987. Homoeobox gene Hox-1.5 expression in mouse embryos: Earliest detection by in situ hybridization is during gastrulation. Development 101: $51-60$.

Holland, P.W.H. and B.L.M. Hogan. 1988a. Expression of the homeobox genes during mouse development: A review. Genes Dev. 2: 773-782.

Holland, P.W.H. and B.L.M. Hogan. 1988b. Spatially restricted patterns of expression of the homeobox-containing gene Hox 2.1 during mouse embryogenesis. Development 102: $159-174$.

Jackobovits, A., G.M. Shackleford, H.E. Varmus, and G.R. Martin. 1986. Two proto-oncogenes implicated in mammary carcinogenesis, int-1 and int-2, are independently regulated during mouse development. Proc. Natl. Acad. Sci. 83: $7806-7810$.

Joyner, A.L. and G.R. Martin. 1987. En-1 and En-2, two mousegenes with sequence homology to the Drosophila engrailed gene: Expression during embryogenesis. Genes Dev. 1: 2938.

Joyner, A.L., T. Kornberg, K.G. Coleman, D.R. Cox, and G.R.Martin. 1985. Expression during embryogenesis of a mouse gene with sequence homology to the Drosophila engrailed gene. Cell 43: 29-37.

Kornberg, T. 1981. engrailed: A gene controlling compartment and segment formation in Drosophila. Proc. Natl. Acad. Sci. 78: 1095-1099.

Kornberg, T., I. Siden, P. O'Farrell, and M. Simon. 1985. The engrailed locus of Drosophila: In situ localization of transcripts reveals compartment-specific expression. Cell 40: 45-53.

Lawrence, P.A. and G. Morata. 1976. Compartments in the wing of Drosophila: A study of the engrailed gene. Dev. Biol. 50: 321-337.

Le Mouellic, H., H. Condamine, and P. Brulet. 1988. Pattern of transcription of the homeo gene Hox-3.1 in the mouse embryo. 1988. Genes Dev. 2: 125-135.

Nieuwkoop, P.D., A.G. Johnen, and B. Albers. 1985. The epigenetic nature of early chordate development, pp. 155-161. Cambridge University Press, Cambridge.

Rijsewijk, F., M. Schuermann, E. Wagenaar, P. Parren, D. Weigeland, and R. Nusse. 1987. The Drosophila homolog of the mouse mammary oncogene int-1 is identical to the segment polarity gene wingless. Cell 50: 649-657.

Shackleford, G.M. and H.E. Varmus. 1987. Expression of the proto-oncogene int-1 is restricted to postmeiotic male germ cells and the neural tube of mid-gestational embryos. Cell 50: $89-95$.

Toth, L.E., K.L. Slawin, J.E. Pintar, and M.C. Nguyen-Huu. 1987. Region-specific expression of mouse homeobox genes in the embryonic mesoderm and central nervous system. Proc. Natl. Acad. Sci. 84: 6790-6794.

Wilkinson, D.G., J.A. Bailes, and A.P. McMahon. 1987. Expression of the proto-oncogene int-1 is restricted to specific neural cells in the developing mouse embryo. Cell 50: 7988. 


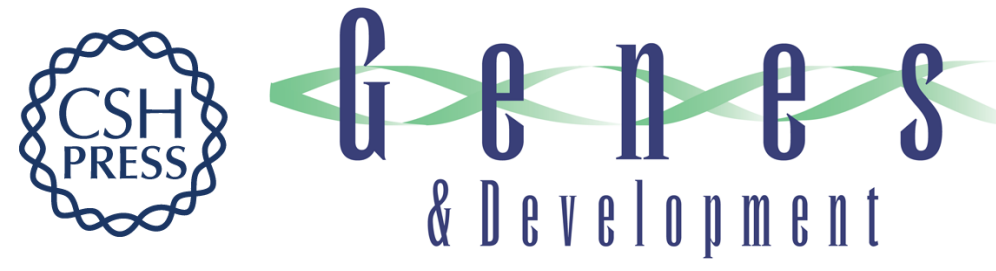

\section{Expression patterns of the homeo box-containing genes En-1 and En-2 and the proto-oncogene int-1 diverge during mouse development.}

C A Davis and A L Joyner

Genes Dev. 1988, 2:

Access the most recent version at doi:10.1101/gad.2.12b.1736

References This article cites 24 articles, 9 of which can be accessed free at:

http://genesdev.cshlp.org/content/2/12b/1736.full.html\#ref-list-1

License

Email Alerting Receive free email alerts when new articles cite this article - sign up in the box at the top Service right corner of the article or click here.

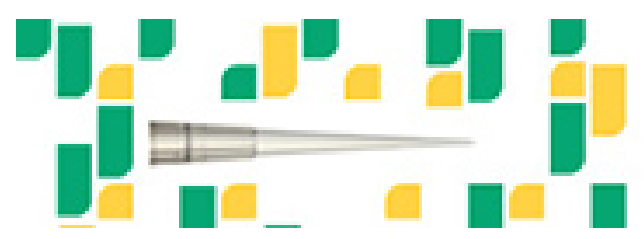

Focused on your science. 